\title{
Effect of heparanase inhibitor on tissue factor overexpression in platelets and endothelial cells induced by anti- $\beta 2-G P I$ antibodies
}

\author{
Antonella Capozzi ${ }^{1}$ | Gloria Riitano ${ }^{1}$ | Serena Recalchi ${ }^{1}$ | Valeria Manganelli ${ }^{1}$ | \\ Roberta Costi $^{2}$ | Francesco Saccoliti ${ }^{2}$ | Fabio Pulcinelli ${ }^{1}$ | Tina Garofalo ${ }^{1}$ | \\ Roberta Misasi $^{1}$ | Agostina Longo ${ }^{1}$ | Roberto Di Santo ${ }^{2}$ | Maurizio Sorice ${ }^{1}$
}

${ }^{1}$ Department of Experimental Medicine,

"Sapienza" University of Rome, Rome, Italy

${ }^{2}$ Dipartimento di Chimica e Tecnologie del Farmaco, Istituto Pasteur-Fondazione Cenci Bolognetti, "Sapienza" University of Rome, Rome, Italy

\section{Correspondence}

Maurizio Sorice, Department of Experimental Medicine-"Sapienza" University of Rome, Viale Regina Elena 324, 00161 Rome, Italy.

Email: maurizio.sorice@uniroma1.it

Funding information

This research was supported by a grant from University of Rome "La Sapienza," Italy (Progetti di Ricerca di Ateneo, 0000055_19 RS_Sorice_RicScient_ progGrandi2019).

\begin{abstract}
Background: Anti-phospholipid syndrome (APS) is characterized by arterial and/or venous thrombosis and pregnancy morbidity associated with the presence of "antiphospholipid antibodies." Thrombosis may be the result of a hypercoagulable state related to activation of endothelial cells and platelets by anti- $\beta 2$-glycoprotein I ( $\beta 2$ GPI) antibodies. Anti- $\beta 2$-GPI antibodies induce a proinflammatory and procoagulant phenotype in these cells that, after activation, express tissue factor (TF), the major initiator of the clotting cascade, playing a role in thrombotic manifestations. Moreover, TF expression may also be induced by heparanase, an endo- $\beta$-D-glucuronidase, that generates heparan sulfate fragments, regulating inflammatory responses.

Objectives: In this study we analyzed, in human platelets and endothelial cells, the effect of a new symmetrical 2-aminophenyl-benzazolyl-5-acetate derivative (RDS3337), able to inhibit heparanase activity, on signal transduction pathways leading to TF expression triggered by anti- $\beta 2-\mathrm{GPI}$.

Methods: Platelets and endothelial cells were incubated with affinity purified anti32-GPI after pretreatment with RDS3337. Cell lysates were analyzed for phosphointerleukin-1 receptor-associated kinase 1 (IRAK1), phospho-p65 nuclear factor kappa $\mathrm{B}(\mathrm{NF}-\kappa \mathrm{B})$ and TF by western blot. In addition, platelet activation and secretion by ATP release dosage were evaluated.

Results: IRAK phosphorylation and consequent NF- $\mathrm{kB}$ activation, as well as TF expression triggered by anti- $\beta 2$-GPI treatment were significantly prevented by previous pretreatment with RDS3337. In the same vein, pretreatment with RDS3337 prevented platelet aggregation and ATP release triggered by anti- $\beta 2$ - $\mathrm{GPI}$ antibodies.

Conclusion: These findings support the view of heparanase involvement in a prothrombotic state related to APS syndrome, suggesting a novel target to regulate overexpression of procoagulant protein(s).
\end{abstract}


KEYWORDS

anti- $\beta 2$-glycoprotein I, anti-phospholipid syndrome, endothelial cells, heparanase inhibitor, platelets, tissue factor

\section{1 | INTRODUCTION}

Anti-phospholipid antibodies (aPL), which include anti- $\beta 2$ glycoprotein I ( $\beta 2-\mathrm{GPI})$, anticardiolipin antibodies ( $\mathrm{aCL})$, and/or lupus anticoagulant (LAC) are serological markers of anti-phospholipid syndrome (APS), a systemic autoimmune disease characterized by clinical features including arterial and/or venous thrombosis, early miscarriages, or fetal deaths. ${ }^{1-3}$ aPL represent a heterogeneous family of antibodies, including anti- $\beta 2-\mathrm{GPI}{ }^{3}$

Anti- $\beta 2$-GPI antibodies may be responsible for thrombosis resulting from a hypercoagulable state related to the activation of endothelial cells and platelets. Indeed, anti- $\beta 2-G P I$ antibodies induce a proinflammatory and procoagulant phenotype in these cells which, after activation, express tissue factor (TF), the main initiator of the coagulation cascade. ${ }^{4}$ It is already known that the dysfunction of endothelial cells and platelets can play an active role in the pathogenesis of deep vein thrombosis and therefore of APS. In fact, the loss of the glycocalyx, a thin layer rich in glycosaminoglycans (GAG) on the surface of endothelial cells, is a key feature of endothelial dysfunction and increases the exposure of adhesion molecules, such as selectins, which are involved in platelet binding to endothelial cells. ${ }^{5}$ Moreover, it was reported that the anti- $\beta 2-\mathrm{GPI} /$ $\beta 2$-GPI complex binds to the platelet thrombus and amplifies platelet activation. In the same paper the authors showed that inhibition of platelet activation prevents the activation of endothelial cells and the formation of fibrin. ${ }^{6}$ Recently, we showed that platelets can express TF on their surface. In particular, it was shown that resting unstimulated platelets express TF and this protein is enhanced or induced following cell activation by a signal transduction pathway that involves interleukin-1 receptor-associated kinase 1 (IRAK) phosphorylation and nuclear factor kappa B (NF-кB) activation. ${ }^{7}$ Furthermore, platelets from APS patients showed a significantly increased expression of TF. ${ }^{7}$ It supported the view that platelets play an important role in the pathogenesis of APS, by activating a signal transduction pathway leading to the release of different procoagulant mediators, as in nucleated cells., ${ }^{8,9}$

Previous data indicate that TF expression may be also induced by heparanase, which is expressed at high levels in placenta, mast cells, neutrophils, lymphocytes, and platelets. Heparanase is an endo- $\beta-D-$ glucuronidase capable of cleaving heparan sulfate (HS) side chains, both in extracellular space and within the cells, regulating several biological activities. Indeed, in addition to its well-characterized role in cancer, heparanase activity may play a role in the pathogenesis of several inflammatory disorders, promoting migration of vascular endothelial cells and activation of immune system cells. ${ }^{10,11}$ Heparanase generates soluble HS fragments that control inflammatory responses at multiple levels, including the release of cytokines/chemokines in the

\section{Essentials}

- In antiphospholipid syndrome anti- $\beta 2$-glycoprotein I ( $\beta 2$ GPI) antibodies induce a signal transduction pathway that leads to tissue factor (TF) expression on the cell surface.

- We analyze the effect of a new heparanase inhibitor on signal transduction pathways leading to TF expression triggered by anti- $\beta 2-\mathrm{GPI}$ in platelets and endothelial cells.

- Signal transduction pathways leading to TF expression as well as platelet aggregation induced by anti- $\beta 2-\mathrm{GPI}$ are shown to be prevented by heparanase inhibitor RDS3337.

- These findings suggest a "new" potential therapeutic target to regulate overexpression of procoagulant protein(s) in antiphospholipid syndrome.

extracellular space, modulation of leukocyte interactions with endothelial cells and extracellular matrix, and initiation of innate immune responses through interactions with Toll-like receptor 4 (TLR-4). ${ }^{12-14}$ This mechanism was demonstrated by the significant inhibition of cytokine release using inhibitors of heparanase enzymatic activity. It revealed that heparanase-induced cytokine release was dependent on MyD88, and thus mediated through TLR-4 via the NF-KB pathway. ${ }^{15,16}$

Thereafter, heparanase, besides being recognized as a proinflammatory protein, is involved in wound healing and in activation of the coagulation cascade. It may act as a cofactor of TF and not only upregulates TF expression in endothelial cells, but also directly interacts with cell surface TF and enhances factor Xa production with subsequent activation of the coagulation system. ${ }^{17}$ The hemostatic function of heparanase suggests a potential clinical relevance of this protein; in fact, targeting the procoagulant domain of heparanase may be useful for patients with pro-angiogenic and prothrombotic conditions. ${ }^{18}$

Over the years, several inhibitors have been discovered searching molecules able to interfere with the biological activity of heparanase; among these, it is possible to find inhibitors of synthetic and natural origin, several heparin derivatives and polysulfated oligosaccharides, proteins, monoclonal antibodies, and nucleic acids. ${ }^{19}$

The development of some inhibitors of heparanase may exceed some of the limitations of polysaccharides. Due to their properties, to be properly designed to have favorable pharmacokinetic and oral availability, small molecules are particularly desirable. Some benzoxazole derivates, in the group of synthetic small molecules, have been described as heparanase inhibitors; among these benzoxazol-5-yl acetic acid showed promising properties as heparanase inhibitors. ${ }^{20,21}$ 
In the present study we describe the biological effect of a new symmetrical 2-aminophenyl-benzazolyl-5-acetate derivative able to inhibit heparanase enzymatic activity.

In particular, we investigate the inhibitory role of this new compound on endothelial cells and platelets activation triggered by anti$\beta 2-\mathrm{GPI}$ antibodies. Because heparanase plays a role in thrombosis and endothelial dysfunction, but also through the enzymatic cleavage of HS, we analyze this specific heparanase inhibitor as a molecule affecting the increase of TF induced by anti- $\beta 2-\mathrm{GPI}$ antibodies. Thus, we may suggest a novel target to regulate overexpression of procoagulant protein(s) (i.e., TF) and, consequently, the prothrombotic state commonly associated with the pathogenesis of thrombosis during APS.

\section{2 | METHODS}

\subsection{Analysis of the effect of heparanase inhibitors on endothelial cell viability}

Human umbilical vein endothelial cells (HUVECs) were grown in PromoCell growth medium containing endothelial cell growth medium kit (PromoCell) and 10\% fetal bovine serum (Sigma-Aldrich), at $37^{\circ} \mathrm{C}$ in a humified $5 \% \mathrm{CO}_{2}$ atmosphere.

The benzazolyl derivatives endowed with potent antiheparanase activity have been designed and developed as previously reported by some authors of this work. ${ }^{19,21}$ Iterative cycles of design, enzymatic tests on heparanase recombinant enzyme, and in vitro cellular assays have been implemented to obtain valuable information for drug design within the hit-to-lead discovery process. On base of dose causing 50\% inhibition of heparanase enzymatic activity, as determined from dose response curves (mean of duplicates; standard deviation [SD] always <10\%), RDS3333, RDS3298, RDS3337, and RDS3098 have been selected for the present work. RDS3333, RDS3298, RDS3337, and RDS3098 correspond to 13a $\left(I C_{50}=0.64 \mu \mathrm{M}\right), 7 \mathrm{~b}\left(I C_{50}=0.37 \mu \mathrm{M}\right), 7 \mathrm{~g}\left(I C_{50}=0.08 \mu \mathrm{M}\right)$, and $5 \mathrm{c}$ $\left(I C_{50}=0.18 \mu \mathrm{M}\right)$, respectively, as reported following the numbering of the references 19 and 21 . Compounds needed for the experiments have been resynthesized as reported ${ }^{19,21}$ obtained with the appropriate purity (>95\%) and supplied in amount sufficient for the assays of this work.

The compounds RDS3333, RDS3298, RDS3337, and RDS3098 were dissolved in dimethyl sulfoxide (DMSO, Sigma-Aldrich) at the 10-mM stock solution. Cells were seeded into 6-well cell culture plates at the concentration of $2 \times 10^{5}$ cells $/ \mathrm{mL}$ and maintained for $24 \mathrm{~h}$ at $37^{\circ} \mathrm{C}$ with $5 \% \mathrm{CO}_{2}$. Then, cells were treated with different concentrations of compounds $(80,320,1280 \mathrm{nM})$ for an incubation time of 24,48 , or $72 \mathrm{~h}$. Vehicle-treated cells or cells incubated with each of the four compounds were analyzed by Trypan Blue (SigmaAldrich) assay to evaluate cell viability. ${ }^{22}$ DMSO is the vehicle to dissolve the compounds and we consider cells without any treatment, with only DMSO, as vehicle-treated cells.

\section{2 | Purification of anti-ק2-GPI antibodies}

Isolation of human anti- $\beta 2-\mathrm{GPI}$ antibodies was obtained by affinity chromatography, as previously reported, ${ }^{8}$ from three APS patients (positive for anti- $\beta 2$-GPI antibodies by ELISA). The three APS patients were women (ages 42, 42, and 44 years) with deep venous and arterial thromboses who had been diagnosed according to the Sidney Classification Criteria. $^{2}$ Antibody reactivity to $\beta 2-\mathrm{GPI}$ was checked by western blot. The purified antibodies recognize the DI domain of $\beta 2$-GPI. They showed abnormal values in at least two clotting tests that returned to normal values on confirmatory testing, displaying LAC activity. In all performed tests, the stimulatory effect of the three anti- $\beta 2-G P I$ antibodies was virtually the same (data not shown). As a control, we used IgG from human normal serum (Sigma-Aldrich).

\subsection{Platelet separation}

Blood samples were obtained from six healthy donors that gave written informed consent from Transfusional Center of Policlinico, Sapienza University of Rome.

For platelet separation, samples in the presence of sodium citrate as anticoagulant were centrifuged at $150 \mathrm{~g}$ for $15 \mathrm{~min}$ at $20^{\circ} \mathrm{C}$ to obtain platelet-rich plasma (PRP). Two thirds of the PRP were drawn, without disturbing the buffy coat layer, to prevent contamination. PRP, transferred into another new sterile tube, was mixed with acid citrate dextrose (ACD) to avoid platelet activation, and centrifuged at $900 \mathrm{~g}$ for $10 \mathrm{~min}$ at $20^{\circ} \mathrm{C}$ (with no brake applied). ${ }^{23}$

After discard platelet-poor plasma (PPP), platelet pellets were resuspended with calcium-free Tyrode's buffer, containing $10 \%$ ( $v: v)$ ACD and washed as above. Then, platelets were resuspended in calcium-free Tyrode's buffer with the addition of bovine serum albumin (BSA, $3 \mathrm{mg} / \mathrm{ml}$ ), previously tested as $\beta 2$-GPI-free by highperformance liquid chromatography (HPLC).

A hemocytometer (Coulter, Beckman Coulter) was used to count the platelets, which revealed that leukocyte contamination was $<1$ leukocyte $/ 10^{7}$ platelets. Then, the purity of the isolated platelets was verified and confirmed by staining with a fluorescein isothiocyanate (FITC)-conjugated anti-CD41 or anti-CD61 antibody (Beckman Coulter) and analyzed by flow cytometry (Coulter Epics, Beckman Coulter; data not shown).

\section{4 | In vitro incubation of endothelial cells and human platelets with anti- $\beta 2-\mathrm{GPI}$ in the presence of heparanase inhibitor}

For in vitro studies, HUVECs $\left(5 \times 10^{5} / \mathrm{mL}\right.$ ), HMEC-1 (American Type Culture Collection, ATCC; $5 \times 10^{5} / \mathrm{mL}$, see supporting information) and human platelets $\left(3 \times 10^{8} / \mathrm{mL}\right)$ were seeded into 6-well cell culture and incubated at $37^{\circ} \mathrm{C}$, for different incubation times, with affinity-purified or polyclonal anti- $\beta 2$-GPI antibodies $(200 \mu \mathrm{g} / \mathrm{ml})$, normal human serum 
IgG $(200 \mu \mathrm{g} / \mathrm{ml})$, or lipopolysaccharide (LPS) (100 ng/ml), according to the methods previously described. ${ }^{7,24}$ To exclude the possibility of LPS contamination, samples were stimulated in the presence or absence of anti- $\beta 2 \mathrm{GPI}$ antibody and then pretreated with polymyxin $B$ (10 $\mu \mathrm{g} / \mathrm{ml}$; Sigma-Aldrich). In parallel experiments, HUVECs, as well as human platelets, were pretreated with selective heparanase inhibitor RDS3337 ( $320 \mathrm{nM}$ ) for $1 \mathrm{~h}$ before treatments. Virtually no heparanase activity was detected in HUVECs and platelets pre-incubated with RDS3337, as detected by Heparanase Assay kit (Amsbio). On the contrary, affinity-purified as well as LPS induced a significant increase of heparanase activity and release (Figure S1 in supporting information).

All materials contained $<0.00025 \mathrm{ng}$ of endotoxin/ $\mu \mathrm{g}$ of protein, as determined by the Limulus amebocyte lysate test (Associates of Cape Cod).

\subsection{Preparation of cell extracts}

Untreated or treated HUVECs and HMEC-1 with affinity-purified anti- $\beta 2$-GPI antibodies, polyclonal anti- $\beta 2-G P I$ antibodies, normal human serum IgG or LPS, and alternatively pretreated with heparanase inhibitor RDS3337, were incubated for different incubation times, at $37^{\circ} \mathrm{C}$, in $5 \% \mathrm{CO}_{2}$. After treatments the medium was removed, cells placed on ice, washed once in phosphate-buffered saline (PBS), and scraped in PBS. To prepare whole-cell extracts, cells were resuspended in lysis buffer, containing 20 mM HEPES, pH 7.2; $1 \%$ Nonidet P-40, $10 \%$ glycerol, $50 \mathrm{mM} \mathrm{NaF}, 1 \mathrm{mM} \mathrm{Na}_{3} \mathrm{VO}_{4}$ and protease inhibitors cocktail (Sigma-Aldrich). Soluble proteins were recovered after centrifugation of lysates at $15,000 \mathrm{~g}$ for $15 \mathrm{~min}$ at $4^{\circ} \mathrm{C}$ Nuclear extracts were prepared as previously described. ${ }^{25}$ Briefly, cells were resuspended in buffer A (20 mM HEPES, pH 7.2; 0.1\% Nonidet P-40, $20 \mathrm{mM} \mathrm{KCl}, 3.0 \mathrm{mM} \mathrm{MgCl}, 1 \mathrm{mM} \mathrm{Na}_{3} \mathrm{VO}_{4}, 5 \mathrm{mM}$ DTT, and protease inhibitors cocktail), after $30 \mathrm{~min}$ on ice, and then centrifuged for $30 \mathrm{~min}$ at $10,000 \mathrm{~g}$ at $4^{\circ} \mathrm{C}$. Pellets were resuspended in buffer $\mathrm{B}$ (40 mM HEPES, pH 7.2; $0.84 \mathrm{M} \mathrm{NaCl}, 0.4$ mM EDTA, 50\% glycerol, $1 \mathrm{mM} \mathrm{Na}_{3} \mathrm{VO}_{4}, 5 \mathrm{mM} \mathrm{DTT}$, and protease inhibitors cocktail), after $1 \mathrm{~h}$ on ice, samples were centrifuged at $10,000 \mathrm{~g}$ for $1 \mathrm{~h}$ at $4^{\circ} \mathrm{C}$ and supernatants (nuclear extracts) were transferred to new vials.

Human platelets, unstimulated or stimulated with either affinitypurified or polyclonal anti- $\beta 2-\mathrm{GPI}$ antibodies, normal human serum IgG, or LPS, and alternatively pretreated with heparanase inhibitor RDS3337, were incubated for different incubation times at $37^{\circ} \mathrm{C}$ in $5 \% \mathrm{CO}_{2}$. Then, samples were resuspended in lysis buffer and wholecell extracts were obtained as described above.

Protein content was determined by Bradford assay using BSA as a standard (Bio-Rad). Samples were frozen at $-80^{\circ} \mathrm{C}$.

\subsection{Western blot analysis of phospho-IRAK1 and phospho-NF-кB}

Equal amounts of whole ( $40 \mu \mathrm{g} / \mathrm{sample})$ or nuclear $(20 \mu \mathrm{g} / \mathrm{sam}$ ple) extract proteins of HUVECs or HMEC-1 and whole extracts proteins (40 $\mu \mathrm{g} / \mathrm{sample})$ of human platelets, untreated or treated with either affinity-purified or polyclonal anti- $32-\mathrm{GPI}$ antibodies, normal human serum IgG, or LPS (HUVECs for $45 \mathrm{~min}$ and platelets for $10 \mathrm{~min}$ ), and alternatively pretreated with heparanase inhibitor RDS3337 for $1 \mathrm{~h}$, were separated in 7.5\% sodium dodecylsulfate polyacyrlamide gel electrophoresis (SDS-PAGE) under unreducing conditions. Proteins were electrophoretically transferred to polyvinylidene difluoride (PVDF) membranes (Bio-Rad Laboratories) and then, after blocking with Tris-buffered saline Tween 20 (TBS-T) 3\% BSA, incubated with polyclonal rabbit anti-phospho-IRAK1 (Cell Signaling, Inc.) or polyclonal rabbit anti-phospho-NF-кB-p65 antibodies (Cell Signaling, Inc.). Antibody reactions were visualized by horseradish peroxidase (HRP)-conjugated anti-rabbit IgG (SigmaAldrich), and then by the chemiluminescence reaction using enhanced chemiluminescence (ECL) western blot system (Amersham Pharmacia Biotech).

To adjust for total protein content, phospho-IRAK1-blotted membranes were stripped and reprobed with polyclonal anti-IRAK1 antibody (MBL) or anti- $\beta$-actin $\mathrm{mAb}$ (Sigma-Aldrich). As a control for loading and purity of preparation, phospho-NF-kB-p65-blotted membranes of HUVECs and HMEC-1 were reprobed with polyclonal anti-histone $\mathrm{H} 1$ antibodies (Abcam) whereas for loading control, phospho-NF-kB-p65-blotted membranes of human platelets were stripped and reprobed with anti-NF-kB-p65 (Cell Signaling, Inc.) or with anti- $\beta$-actin mAb (Sigma-Aldrich).

Densitometric scanning analysis was performed by NIH Image 1.62 software (National Institutes of Health). The density of each band (absolute value) in the same gel was analyzed.

\section{7 | Analysis of TF expression}

Preliminarily, TF expression on platelets was analyzed by immunofluorescence. Unstimulated human platelets or stimulated with affinity-purified anti- $\beta 2-\mathrm{GPI}$ antibodies for $45 \mathrm{~min}$ at $37^{\circ} \mathrm{C}$ were dried-fixed on glass slides. Then, platelets were fixed with $4 \%$ paraformaldehyde in PBS for $30 \mathrm{~min}$ at room temperature and then permeabilized by $0.5 \%$ Triton X-100 in PBS for 5 min at room temperature. After washing nonspecific binding sites were blocked with PBS containing 3\% BSA. Platelet CD61 and TF were immunolabeled with FITC-conjugated anti-CD61 mAb (Beckman Coulter) or with FITC-conjugated anti-TF mAb (Sino Biological). The slides were examined by fluorescence microscopy. The images were acquired and observed with an Olympus BX51 fluorescence microscope (Olympus Italia S.r.l.; $\times 63$ magnification) equipped with an F- View digital camera and the Cell-F Digital Imaging Software.

In addition, modulation of TF expression was analyzed by western blot analysis. Equal amounts of proteins lysates ( $40 \mu \mathrm{g} / \mathrm{sample})$ of HUVECs and human platelets (from untreated or treated with affinity-purified anti- $\beta 2$-GPI antibodies, normal human serum IgG, or LPS for $4 \mathrm{~h}$, and alternatively pretreated with heparanase inhibitor RDS3337 for $1 \mathrm{~h}$ ) were subjected to SDS-PAGE on $10 \%$ gels and then electrophoretically transferred to PVDF membranes. The 
membranes were blocked with TBS-T containing 3\% BSA and subsequently probed with rabbit anti-TF mAb (Abcam). Bound antibodies were visualized with HRP-conjugated anti-mouse IgG, and immunoreactivity was assessed by ECL reaction using the $E C L$ western blot system. For loading control TF-blotted membranes were reprobed with anti- $\beta$-actin $\mathrm{mAb}$ (Sigma-Aldrich).

\section{\begin{tabular}{l|l}
2.8 & Analysis of TF procoagulant activity
\end{tabular}}

A two-stage factor $X$-activating assay ${ }^{26}$ was used to analyze the procoagulant activity of TF released in supernatant of platelets treated with affinity-purified anti- $\beta 2-\mathrm{GPI}$ antibodies $(200 \mu \mathrm{g} / \mathrm{ml})$, normal human serum IgG $(200 \mu \mathrm{g} / \mathrm{ml})$, or LPS $(100 \mathrm{ng} / \mathrm{ml})$, in presence or not of heparanase inhibitor RDS3337 (320 nM, 1 h before treatments). Briefly, platelets from normal donors were stimulated in TBS containing $0.1 \%$ BSA and supernatants were collected. A fixed amount of proteins $(100 \mu \mathrm{g})$ released from platelets was incubated in triplicate with activated factor VII (FVIla; $10 \mathrm{ng}$ ) and $\mathrm{CaCl}_{2}(5 \mathrm{mM})$ for $15 \mathrm{~min}$ at $37^{\circ} \mathrm{C}$. Then we added human factor $X(250 \mathrm{ng})$ and the mixture was reincubated for $30 \mathrm{~min}$ at $37^{\circ} \mathrm{C}$. Finally, $10 \mu \mathrm{l}$ of FXa-specific chromogenic substrate $(5 \mathrm{mM})$ was added and factor $\mathrm{Xa}$ activity was quantitated by measuring the rate of increase in absorbance at $405 \mathrm{~nm}$ using an ELISA plate reader. All coagulation factors were purchased from Sigma-Aldrich.

\section{9 | Platelet activation}

Platelets from healthy donors were obtained as reported above. Platelets were treated with a mix of agonists composed by U446619 ( $1 \mu \mathrm{M}$; synthetic thromboxane A2 receptor agonist, Helena Biosciences Europe) plus epinephrine $(10 \mu \mathrm{M}$; Helena Biosciences Europe) or affinity-purified anti- $\beta 2-\mathrm{GPI}$ antibodies $(200 \mu \mathrm{g} / \mathrm{ml})$, and alternatively pretreated with heparanase inhibitor RDS3337 for $1 \mathrm{~h}$, were analyzed to evaluate platelet aggregation. Briefly, platelets were added, after treatment, in appropriate wells and the plate, covered with an acrylic adhesive thin layer, was placed immediately in a Plate Reader Victor 3 (PerkinElmer). The absorbance, carrying out an orbital shaking for $10 \mathrm{~s}$, before each reading, was determined at $590 \mathrm{~nm}$ every $60 \mathrm{~s}$ at $37^{\circ} \mathrm{C}$. The platelet aggregation percentage (PA\%) was assessed using the following formula PA\% = (sample UA absorbance-PRP UA absorbance)/(PPP UA absorbance-PRP UA absorbance) $\times 100$.

Platelet function was also investigated evaluating platelet secretion by ATP release dosage. ATP release, post-platelet activation, was quantified using a luciferin/luciferase method (ATP lite, PerkinElmer), as ATP monitoring system. This method was performed as reported. ${ }^{27}$ Briefly, ATPlite, according to the manufacturer's instructions, was added to the platelet suspension immediately after the treatments. The plate was placed in a Plate Reader Victor 3 (PerkinElmer) to measure the emitted light every $60 \mathrm{~s}$ at $37^{\circ} \mathrm{C}$ for $10 \mathrm{~min}$. The results were analyzed as percentage of maximal secretion compared to total intraplatelets adenyl nucleotides content.

\subsection{0 | Statistical analysis}

Data obtained are expressed as means \pm SD of at least six independent experiments. Statistical analysis was performed with two statistical tests. To compare differences between percentages of Trypan Blue positive cells we used Chi-square $\left(\chi^{2}\right)$ tests. In the other experiments the paired Student's $t$-test was used. Statistical significance was set at $P \leq .01 ; P$ values $>.01$ were not significant (NS).

\section{3 | RESULTS}

\subsection{Preliminary analysis of activity and cytotoxic effect of Heparanase inhibitors}

Four newly synthesized compounds were already tested for their ability to inhibit heparanase activity using an in vitro assay. ${ }^{19,21}$ The compound RDS3337 (Figure 1) revealed the highest anti-heparanase activity among the tested compounds, showing nanomolar potency. Indeed, it reported an $I C_{50}$ value of $0.08 \mu \mathrm{M}$, being therefore about 2-8 more potent than its analogues (Table 1 ). These compounds were further studied to assess their effect on cell viability. Thus, we performed a preliminary analysis to exclude their cytotoxic effect, under

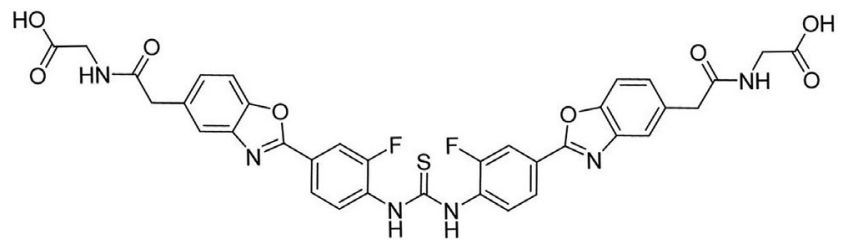

FIGURE 1 Newly synthesized compound RDS3337 that reports anti-heparanase activity

TABLE 1 Heparanase inhibitory activities of the newly synthesized compounds RDS3333, RDS3298, RDS3337, and RDS3098.

\begin{tabular}{ll}
\hline Compound & $\begin{array}{l}I_{50} \\
(\mu \mathrm{M})^{\mathrm{a}}\end{array}$ \\
\hline RDS3333 (13a) & 0.64 \\
\hline RDS3298 (7b) & 0.37 \\
\hline RDS3337 (7g) & 0.08 \\
\hline RDS3098 (5c) & 0.18 \\
\hline
\end{tabular}

${ }^{a}$ Dose causing 50\% inhibition of heparanase enzymatic activity as determined from dose response curves (mean of duplicates; standard deviation always < $10 \%$ ) repeated at least twice in separate experiments, stated in micromole concentration. 
our experimental conditions, using Trypan Blue assay. HUVECs were treated with RDS3333, RDS3298, RDS3337, and RDS3098 (concentration range $80-1280 \mathrm{nM}$ ) for different incubation times (24-72 h) and then analyzed by cell counting (Figure 2). The results indicate that RDS3337 is the most interesting compound; this derivative proved to be the most active to inhibit heparanase enzyme, interfering least with cell viability. Thus, we decided to deepen the role of compound RDS3337, as selective heparanase inhibitor, in biological activity studies.

\section{2 | Heparanase inhibitor RDS3337 decreases endothelial cell activation signaling triggered by anti- 及2-GPI antibodies}

Because heparanase may play a role in endothelial dysfunction, we investigated whether the selective heparanase inhibitor RDS3337 is able to affect the activation of molecules usually involved in a proinflammatory signaling. Several data prove that anti- $\beta 2-G P I$ antibodies can activate endothelial cells leading to a proinflammatory phenotype; thus, we analyzed by western blot IRAK phosphorylation and NF-KB activation. Analysis of cell lysates from HUVECs showed that affinity-purified anti- $\beta 2-$ GPI antibodies, as well as LPS, induced IRAK1 phosphorylation (Figure $3 \mathrm{~A}$ ) and NF-кB-p65 activation (Figure 3B), as revealed, respectively, by anti-phospho-IRAK1 and anti-phospho-NF-кB-p65 antibodies' reactivity. Virtually no activation was obtained in untreated cells or following treatment with control human IgG. On the contrary, IRAK phosphorylation and NF-kB-p65 activation significantly decreased when cells were pretreated with the heparanase inhibitor RDS3337 (Figure 3A,B). Similar findings were obtained after triggering of HMEC-1 with polyclonal anti- $\beta 2-\mathrm{GPI}$ antibodies (Figure S2 in supporting information).

These data support the view of heparanase involvement in endothelial cell activation.

\section{3 | Heparanase inhibitor RDS3337 decreases platelet activation signaling triggered by anti- $\beta 2-$ GPI antibodies}

It is well known that platelets may play a role in thrombotic manifestations by binding of aPL, and are a rich source of heparanase. Starting from these data, we analyzed whether the heparanase inhibitor can prevent platelet activation and the signal transduction pathway triggered by anti- $\beta 2$-GPI antibodies. We analyzed by western blot IRAK phosphorylation and NF- $\mathrm{KB}$ activation in platelet lysates. As expected, the treatment with affinity-purified anti- $\beta 2$ GPI antibodies, as well as LPS, induced an increase of phosphoIRAK1 (Figure 4A) and phospho-NF-кB-p65 expression (Figure 4B) compared to untreated platelets or treated with control human IgG. Interestingly, when platelets were pretreated with the selective heparanase inhibitor RDS3337, both anti-phospho-IRAK1 and anti-phospho-NF-kB-p65 reactivity were significantly inhibited (Figure 4A,B). Similar findings were obtained after triggering with polyclonal anti- $\beta 2$-GPI antibodies (Figure $\mathrm{S} 2$ ), demonstrating a functional role of heparanase enzyme in platelet activity.

\subsection{Heparanase inhibitor RDS3337 decreases TF expression triggered by anti- $\beta 2$-GPI antibodies}

We analyzed preliminary expression and distribution of TF in platelets (Figure 5). Immunofluorescence analysis revealed an uneven distribution of the staining mainly in the cytoplasm. Following treatment with affinity-purified anti- $\beta 2-\mathrm{GPI}$ antibodies, TF staining was increased and appeared also localized on cell plasma membrane (see arrows).

Thus, we analyzed the expression of TF as a procoagulant factor not only in endothelial cells, but also in platelets and investigated the role of the heparanase inhibitor RDS3337 on TF expression.

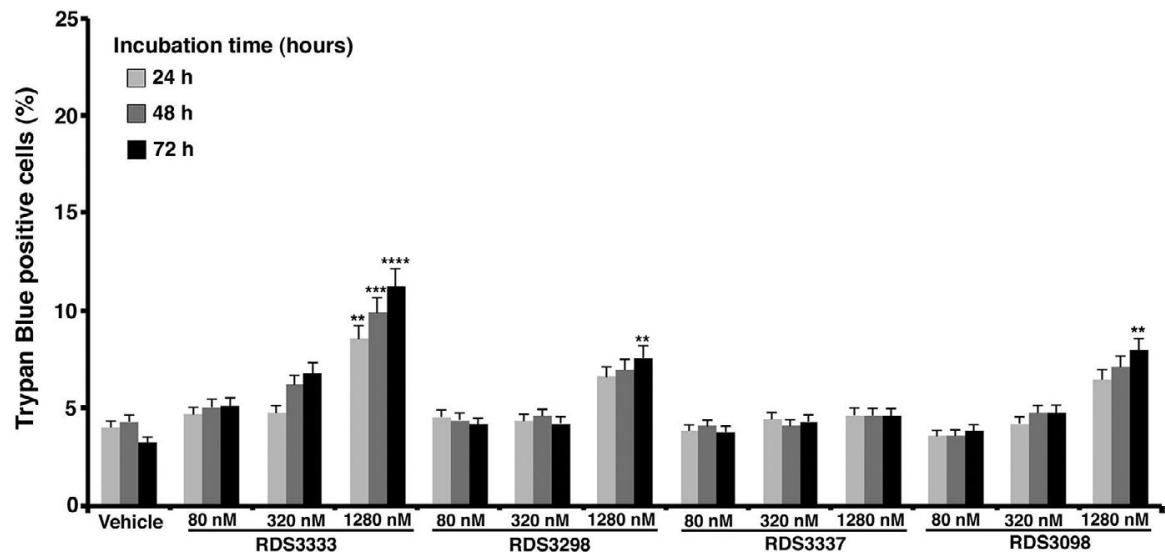

FIGURE 2 Effect on cell viability of newly synthesized compounds RDS3333, RDS3298, RDS3337, and RDS3098. Human umbilical vein endothelial cells were cultured with three different concentrations of the compounds (80, 320, and $1280 \mathrm{nM}$ ) for 24,48 , and $72 \mathrm{~h}$. The number of viable cells was determined by Trypan Blue exclusion test. Data are reported as the mean \pm standard deviation (SD) among 10 independent experiments. Statistical analysis: ${ }^{* *} P<.01$ versus vehicle, ${ }^{* * *} P<.001$ versus vehicle, ${ }^{* * * *} P<.0001$ versus vehicle. RDS3337 treated samples versus vehicle NS, not significant 
A

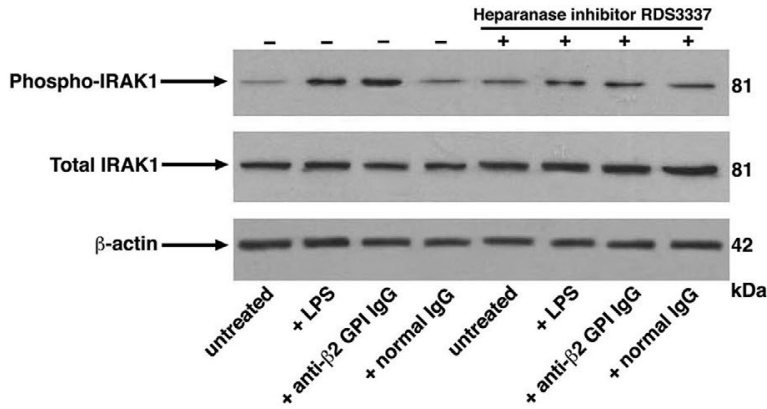

B

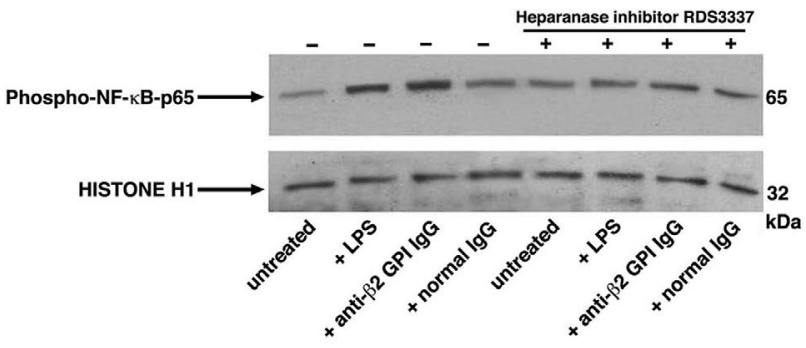

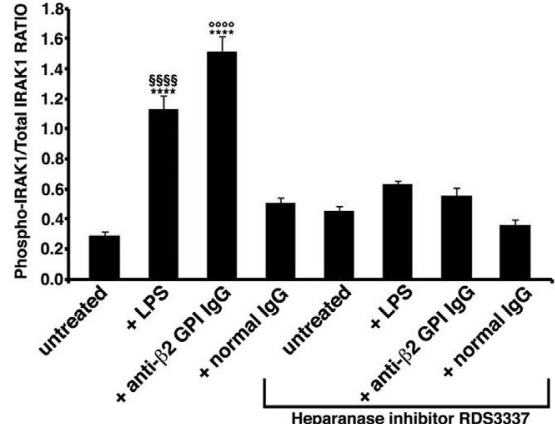

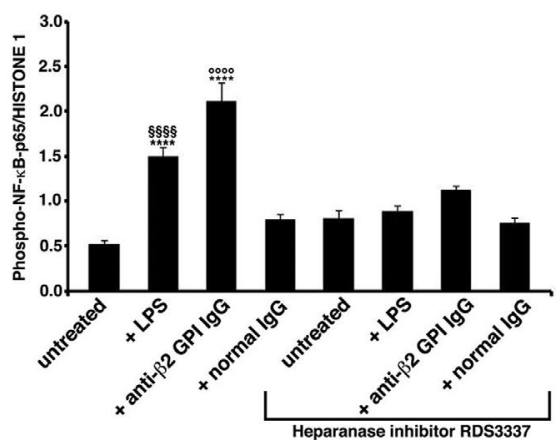

FIGURE 3 Heparanase inhibitor RDS3337 reduces interleukin-1 receptor-associated kinase 1 (IRAK) phosphorylation and nuclear factor kappa B (NF- $\mathrm{B}$ ) activation triggered by anti- $\beta 2$-glycoprotein I ( $\beta 2-\mathrm{GPI})$ antibodies in endothelial cells. Human umbilical vein endothelial cells were treated for $45 \mathrm{~min}$ with affinity-purified anti- $\beta 2-\mathrm{GPI}$ antibodies $(200 \mu \mathrm{g} / \mathrm{ml})$ and, as controls, with lipopolysaccharide (LPS; $100 \mathrm{ng} / \mathrm{ml}$ ) or control human IgG $(200 \mu \mathrm{g} / \mathrm{ml})$. Alternatively, cells were pretreated with heparanase inhibitor RDS3337 (320 nM) for $1 \mathrm{~h}$. After treatments cells were analyzed by western blot for IRAK phosphorylation and NF- $\mathrm{kB}-\mathrm{p} 65$ activation. A, Phosphorylated levels of IRAK1 were evaluated in whole cell extracts using rabbit anti-phospho-IRAK1 Ab. The membrane was stripped and reprobed with polyclonal anti-IRAK1 Ab. For loading control was used anti- $\beta$-actin mAb. Densitometric phospho-IRAK1/Total IRAK1 ratios are shown in the right panel. Results represent the mean \pm SD from six independent experiments. Statistical analysis indicates: ${ }^{* * * *} P<.0001$ versus untreated; ${ }^{\S \S \S \S} P<.0001$ versus RDS3337 + LPS; ${ }^{\circ 000} \mathrm{P}<.0001$ versus RDS3337 + anti- $\beta 2-\mathrm{GPI}$ antibodies. B, Nuclear cell extracts were analyzed to verify phosphoNF-кB-p65 expression using rabbit anti-phospho-NF-кB-p65 Ab. As a control, for loading and purity of preparation, membrane was stripped and reprobed with polyclonal anti-HISTONE H1 Ab. Densitometric phospho-NF-кB-p65/HISTONE H1 ratios are shown in the right panel. Results represent the mean \pm SD from six independent experiments. Statistical analysis indicates: ${ }^{* * * *} P<.0001$ versus untreated;

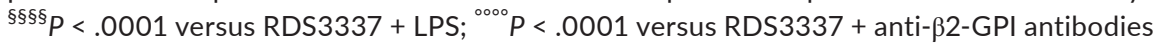

As shown in Figure 5, western blot analysis of endothelial cells and platelet lysates revealed an increase of TF expression following incubation with affinity-purified anti- $\beta 2$-GPI antibodies or LPS, which was reported to induce a significant increase of TF synthesis ${ }^{24}$ as well as of TF expression. ${ }^{28}$ Conversely, virtually no reactivity was observed in untreated cells or in cells stimulated with control human $\operatorname{lgG}$. Furthermore, in samples pretreated with the heparanase inhibitor RDS3337, we obtained a significant decrease of TF levels (Figure 6A,B).

In parallel experiments procoagulant activity of TF was evaluated. As expected, we observed a significant increase $(P<.001)$ of TF activity released from platelets incubated with affinitypurified anti- $\beta 2-\mathrm{GPI}$ antibodies compared to control cells (OD $0.79+0.025$ vs. $0.40+0.035)$, which was partially prevented by previous pretreatment with the heparanase inhibitor RDS3337 (OD $0.45+0.019$ ).

These results suggest an implication of heparanase in determining a prothrombotic state related to endothelial cell injury and platelet activation.

\section{5 | Heparanase inhibitor RDS3337 decreases platelet aggregation and ATP release triggered by

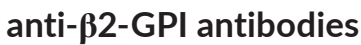

To investigate the effect of heparanase inhibitor on functional platelet activation, platelets from healthy donors were treated with anti- $\beta 2$-GPI antibodies. As shown in Figure 7, an increase of platelet aggregation following incubation with affinity-purified anti- $\beta 2-\mathrm{GPI}$ antibodies was detected, which was decreased in samples pretreated with the heparanase inhibitor RDS3337 (64.4\%).

Moreover, to deepen platelet activation, the secretion of adenyl nucleotides was measured. Results highlighted an increase of ATP release in samples stimulated with anti- $\beta 2$-GPI antibodies, which was partially prevented by previous pretreatment with the heparanase inhibitor RDS3337 (57.1\%).

Alternatively, for both tests (aggregation and ATP release), platelets were treated with a mix of control agonists composed by $U$ 446619 plus epinephrine. In this case, virtually no reduction was observed when cells were pretreated with the heparanase inhibitor 

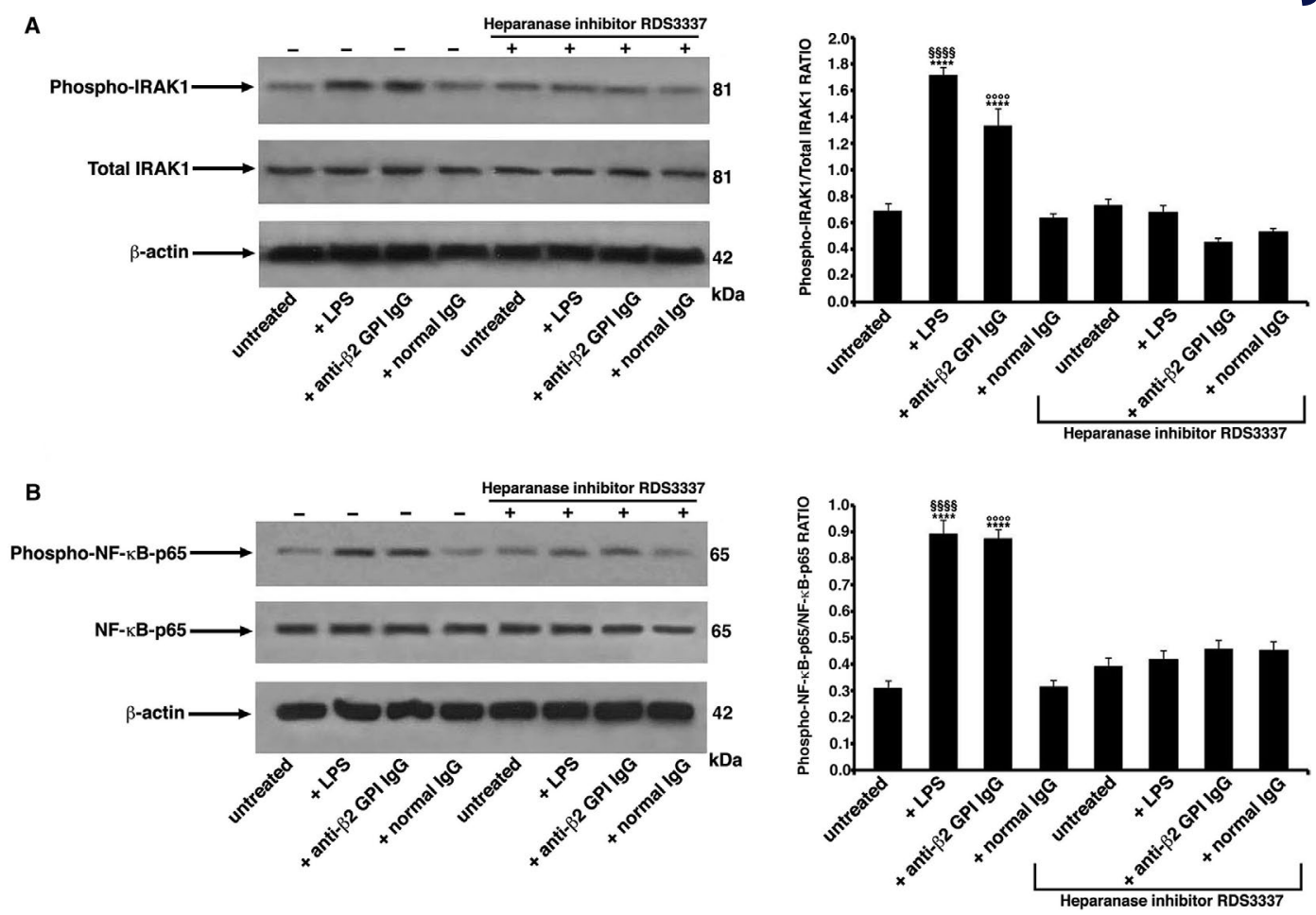

FIGURE 4 Heparanase inhibitor RDS3337 decreases interleukin-1 receptor-associated kinase 1 (IRAK) phosphorylation and nuclear factor kappa B (NF- $\mathrm{kB}$ ) activation triggered by anti- $\beta 2$-glycoprotein I ( $\beta 2$-GPI) antibodies in platelets. Human platelets from healthy donors were treated for $10 \mathrm{~min}$ with affinity-purified anti- $\beta 2-\mathrm{GPI}$ antibodies $(200 \mu \mathrm{g} / \mathrm{ml})$ and, as controls, with lipopolysaccharide (LPS; $100 \mathrm{ng} / \mathrm{ml})$ or control human IgG $(200 \mu \mathrm{g} / \mathrm{ml})$. Alternatively, cells were pretreated with heparanase inhibitor RDS3337 (320 nM) for $1 \mathrm{~h}$. After treatments cells were analyzed by western blot for IRAK phosphorylation and NF- $\mathrm{KB}-\mathrm{p} 65$ activation. A, Whole platelet extracts were used to determine the levels of phospho-IRAK1 expression using rabbit anti-phospho-IRAK1 Ab. The membrane was stripped and reprobed with polyclonal anti-IRAK1 Ab. For loading control was used anti- $\beta$-actin mAb. Densitometric phospho-IRAK1/Total IRAK1 ratios are shown in the right panel. Results represent the mean \pm standard deviation (SD) from six independent experiments. Statistical analysis indicates: ${ }^{* * * *} P<.0001$ versus untreated; ${ }^{\S \S \S} \mathrm{P}<.0001$ versus RDS3337 + LPS; ${ }^{0000} \mathrm{P}<.0001$ versus RDS3337 + anti- $\beta 2$-GPI antibodies. B, Phosphorylated levels of

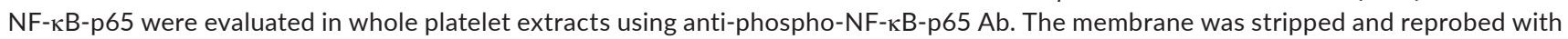

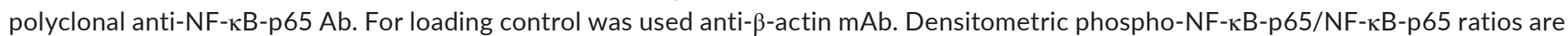
shown in the right panel. Results represent the mean \pm SD from six independent experiments. Statistical analysis indicates: ${ }^{* * * *} P<.0001$ versus untreated; ${ }^{\S \S \S} P<.0001$ versus RDS3337 + LPS; ${ }^{\circ 000} P<.0001$ versus RDS3337 + anti- $\beta 2-$ GPI antibodies

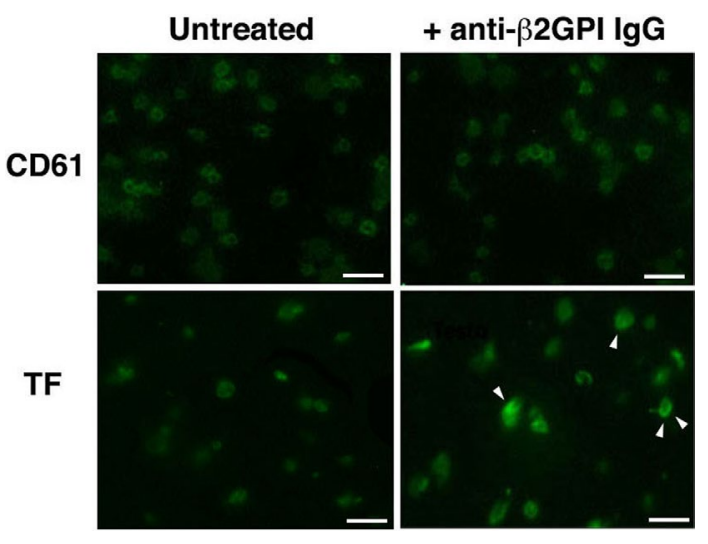

FIGURE 5 Platelet TF expression and distribution. Untreated human platelets and treated with anti- $\beta 2$-glycoprotein I ( $\beta 2$-GPI) for $45 \mathrm{~min}$ at $37^{\circ}$ were fixed with $4 \%$ paraformaldehyde and then permeabilized with $0.5 \%$ Triton X-100. The immunofluorescence pattern of platelets incubated with FITC-conjugated anti-CD61 $\mathrm{mAb}$ or with FITC-conjugated anti-TF mAb is shown. Scale bars: $20 \mu \mathrm{m}$
RDS3337, suggesting that the inhibitory effect of RDS3337 is quite specific.

\section{DISCUSSION}

This study demonstrates that platelet and endothelial cell TF expression induced by human anti- $\beta 2$-GPI antibodies is prevented by heparanase inhibitor. We tested a newly synthesized compound (RDS3337), which was revealed to be highly active without interfering with cell viability. This compound interacts with HBD-1 and HBD-2 regions of heparanase, thus undertaking a high number of polar contacts with the protein counterpart. According to Messore et al. ${ }^{19}$ RDS3337 (7g) may be considered highly specific for heparanase, although we cannot exclude other off-target effects. Moreover, our results showed a significant inhibitory effect on the signal transduction pathway(s) triggered by anti- $\beta 2-\mathrm{GPI}$ antibodies. 
A

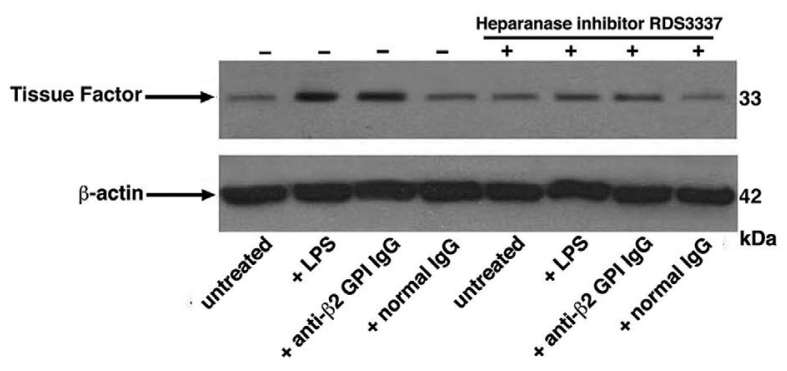

B

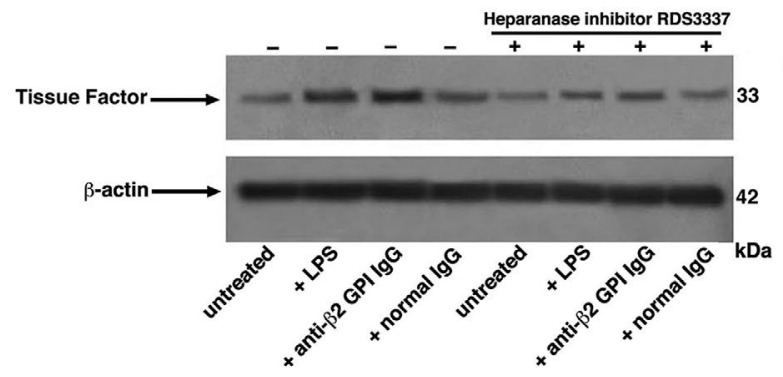

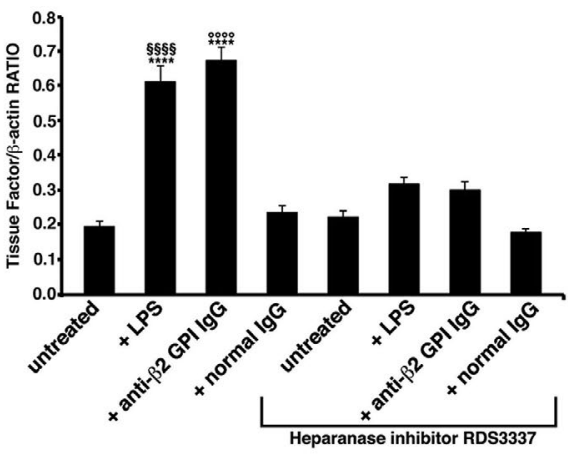

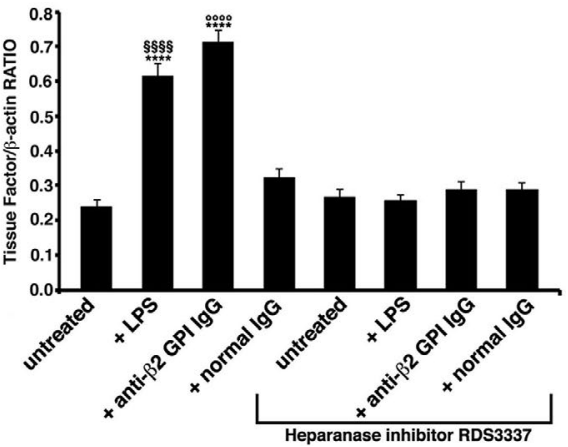

FIGURE 6 Heparanase inhibitor RDS3337 reduces tissue factor (TF) expression triggered by anti- $\beta 2$-glycoprotein I ( $\beta 2$-GPI) antibodies in endothelial cells and platelets. Human umbilical vein endothelial cells and human platelets from healthy donors were treated for $4 \mathrm{~h}$ with affinity-purified anti- $\beta 2-\mathrm{GPI}$ antibodies $(200 \mu \mathrm{g} / \mathrm{ml})$ and, as controls, with lipopolysaccharide (LPS; $100 \mathrm{ng} / \mathrm{ml})$ or control human lgG $(200 \mu \mathrm{g} / \mathrm{ml})$. Alternatively, cells were pretreated with heparanase inhibitor RDS3337 (320 nM) for $1 \mathrm{~h}$. After treatments, whole extracts of endothelial cells (A) and platelets (B) were analyzed by western blot to determine TF expression using rabbit anti-TF mAb. For loading control, membrane was stripped and reprobed with anti- $\beta$-actin mAb. Densitometric TF/ $\beta$-actin ratios are shown in the right panel. Results represent the mean \pm standard deviation (SD) from six independent experiments. Statistical analysis indicates: $(A){ }^{* * * *} P<.0001$ versus untreated; ${ }^{\S \S \S} \mathrm{P}<.0001$ versus RDS3337 + LPS; ${ }^{0000} \mathrm{P}<.0001$ versus RDS3337 + anti- $\beta 2-\mathrm{GPI}$ antibodies. $(\mathrm{B}){ }^{* * * *} \mathrm{P}<.0001$ versus untreated; $\S \S \S \S P<.0001$ versus RDS3337 + LPS; ${ }^{0000} P<.001$ versus RDS3337 + anti- $\beta 2-$ GPI antibodies
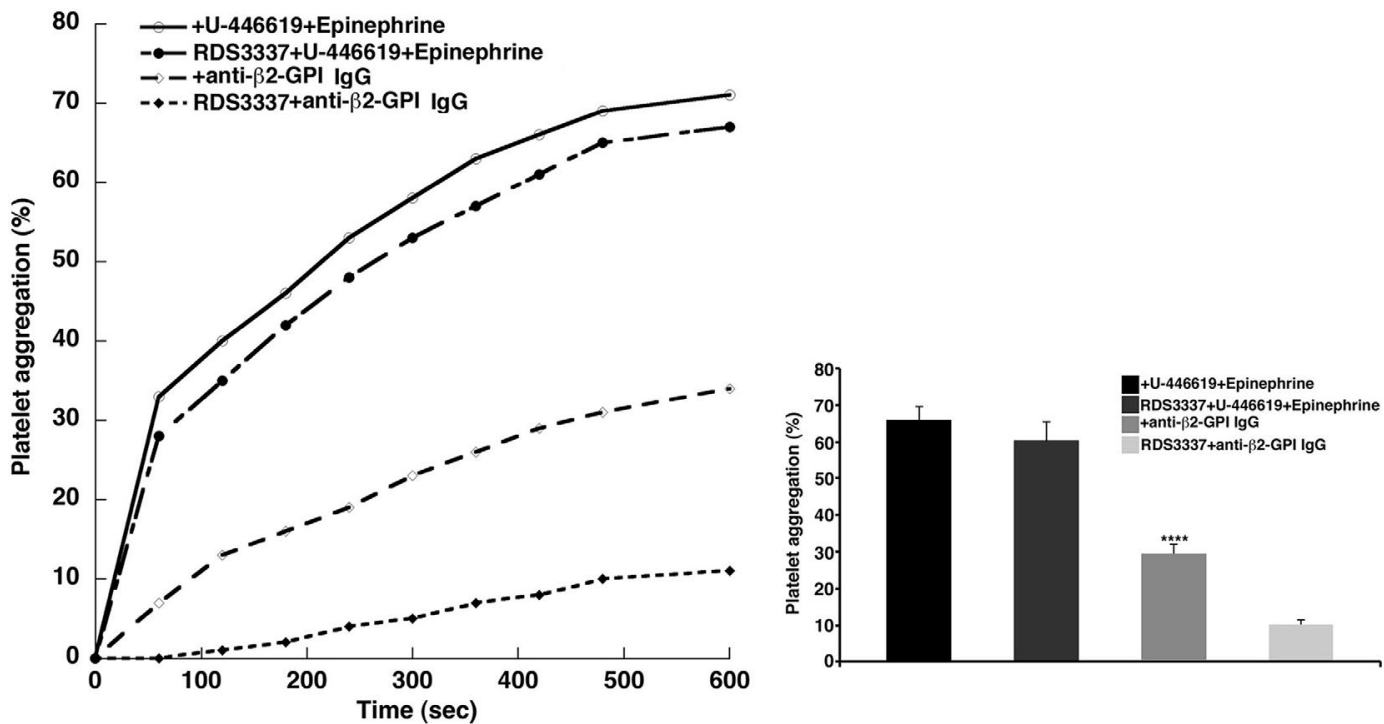

FIGURE 7 Heparanase inhibitor RDS3337 decreases platelet aggregation. Human platelets from healthy donors were treated with a mix of agonists composed by $1 \mu \mathrm{M} \mathrm{U}-446619$ plus $10 \mu \mathrm{M}$ epinephrine or with affinity-purified anti- $\beta 2$-glycoprotein I ( $\beta 2$-GPI) antibodies $(200 \mu \mathrm{g} / \mathrm{ml})$. Alternatively, cells were pretreated with heparanase inhibitor RDS3337 (320 nM) for $1 \mathrm{~h}$. After treatments platelets were analyzed, by a Plate Reader Victor 3, to evaluate platelet aggregation. Results are representative of six independent experiments. Statistical analysis indicates: ${ }^{* * * *} P<.0001$ versus RDS3337 + anti- $\beta 2-\mathrm{GPI}$ antibodies 
In previous studies we demonstrated that these antibodies are able to trigger IRAK phosphorylation and NF-KB translocation, leading to a pro-inflammatory and pro-coagulant monocyte phenotype, characterized by overexpression and release of TF. ${ }^{8}$ Similar findings were also observed in endothelial cells. ${ }^{29}$ Several mechanisms have been proposed for endothelial cell activation by aPL. It may result from the direct binding of $\beta 2-\mathrm{GPI}$ to endothelial cells and the activation of inflammatory receptors on these cells. ${ }^{30}$ Similarly, Laplante et al. ${ }^{31}$ showed in a carotid artery injury model that anti- $\beta 2-$ GPI activation of endothelial cells is dependent on TLR-4. Conversely, anti- $\beta 2-\mathrm{GPI}$ antibodies enhance the production of pro-thrombotic and pro-inflammatory responses in blood vessels. Moreover, antibodies may activate endothelial cells increasing the expression level of von Willebrand factor in their surface, which can contribute to platelet activation. Indeed, recently we reported that anti- $\beta 2-\mathrm{GPI}$ antibodies also induce TF expression in platelets. ${ }^{7}$ The presence of TLR-4 in platelets is currently well known and may play a role in thrombocytopenia induced by LPS. ${ }^{32}$ Indeed, platelets contain all of the proteins (e.g., MyD88, IRAK, and interferon regulatory factor 3 [IRF3]), that are required for signal transduction through TLR-4 and utilize the same mechanisms as in nucleated cells. ${ }^{33}$ Thus, anti- $\beta 2$-GPI triggered activation of the TLR-4 signaling pathway in platelets may play a role in some pathological manifestations of APS. Indeed, TLR-4 is able to trigger a signaling cascade leading to the activation of transcription factors through both MyD88-IRAK-dependent and-independent pathways, with consequent functional effect on platelet activity, hemostasis, and thrombosis. ${ }^{34}$ Moreover, our results showed a significant increase of TF in platelets from APS patients compared to control subjects. ${ }^{7}$

In the present study we confirmed and extended these findings, demonstrating for the first time the in vitro effect of heparanase inhibitors on both TF expression on platelets and endothelial cells and on the signal transduction pathway triggered by anti- $\beta 2-\mathrm{GPI}$ antibodies. The same effect was observed on the signaling triggered by LPS; this observation is not surprising, as it was well known that heparanase activation induces TF expression. ${ }^{17}$ We cannot exclude the possibility that heparanase fragments may induce TF expression on the cells. Anyway, our findings suggest that heparanase activity is released very shortly after anti- $\beta 2-\mathrm{GPI}$ antibody stimulation. Thus, an interplay between heparanase inhibitor and TLR-4 could be hypothesized. However, recent data demonstrated that heparanaseinduced cytokine release was abolished by enzymatic-inhibitors of heparanase. Because soluble heparanase can signal through the TLR pathway, heparanase may also promote upregulation of cytokines through the generation of heparanase-cleaved fragments of the enzyme. The pathway involved in cytokine upregulation was identified as NF-kB-dependent. ${ }^{16}$

Heparanase activity is implicated in neovascularization, inflammation, and autoimmunity. Heparanase expression is enhanced in patients with Crohn's disease and ulcerative colitis and in the synovial fluid and tissue of patients with rheumatoid arthritis. It has also been implicated in the severity of atherosclerosis, because its expression is upregulated in vulnerable coronary plaques. ${ }^{14,35-37}$ This enzyme can play a role as inflammatory mediator, modifying and destroying endothelial surface layer structure, leading to the impairment of endothelial functions, promoting progression of vascular diseases. ${ }^{38}$ Moreover, heparanase induces the transcription of pro-angiogenic, pro-thrombotic, and pro-inflammatory factors, promoting the release of pro-inflammatory cytokines from human peripheral blood mononuclear cells and generating soluble HS fragments that activate TLR-dependent pathway(s). In fact, cytokine induction by heparanase appears to involve TLR-2, TLR-4, and NF-кB. ${ }^{16,17,39}$ The involvement of the enzyme in inflammatory reactions was further supported by anti-inflammatory effects demonstrated for heparanase-inhibiting substances (i.e., heparin, synthetic heparin-mimicking compounds) in animal and clinical studies. Thus, therapeutic benefits are expected in inflammation by pharmacological strategies that target heparanase, reducing heterotypic interactions among epithelial, endothelial, and immune cells due to the enzyme involvement. ${ }^{40-42}$

Heparanase upregulates the expression of TF and interacts with the TF pathway inhibitor (TFPI) on the cell surface membrane of endothelial cells, leading to dissociation of TFPI and increasing coagulation activity. It was also demonstrated that heparanase overexpressing mice generated a larger thrombus within a shorter period of time compared to control mice, suggesting the procoagulant effect of heparanase. ${ }^{18,43}$ In conclusion, demonstration that this new synthesized heparanase inhibitor (RDS3337) is able to prevent the main signal transduction pathway triggered by anti- $\beta 2$-GPI antibodies in endothelial cells and platelets introduces a new task in the pharmacological approach of APS. In addition, heparanase activity detection may be useful in monitoring patients and evaluating risk stratification. Further studies are in progress to test the levels of heparanase activity in APS patients and the relationship with disease progression.

\section{CONFLICTS OF INTEREST}

The authors declare no conflicts of interest.

\section{AUTHOR CONTRIBUTIONS}

M.S., R.D.S., and A.L. conceived and designed the study. G.R. and S.R. performed the experiment; A.C., V.M., and F.S. analyzed the data; A.C., R.M., A.L., and M.S. wrote the original manuscript. M.S., T.G., R.C., and F.P. read and approved the final version of the manuscript.

\section{ETHICAL APPROVAL}

The study was conducted in compliance with the Declaration of Helsinki and the local ethical committee approved this study.

\section{REFERENCES}

1. Cervera R. Antiphospholipid syndrome. Thromb Res. 2017;151:S43-S47.

2. Miyakis S, Lockshin MD, Atsumi T, et al. International consensus statement on an update of the classication criteria for definite antiphospholipid syndrome (APS). J Thromb Haemost. 2006;4: 295-306. 
3. Salmon JE, de Groot PG. Pathogenic role of antiphospholipid antibodies. Lupus. 2008;17:405-411.

4. Bontadi A, Ruffatti A, Falcinelli E, et al. Platelet and endothelial activation in catastrophic and quiescent antiphospholipid syndrome. Thromb Haemost. 2013;109:901-908.

5. Wodicka JR, Chambers AM, Sangha GS, Goergen CJ, Panitch A. Development of a glycosaminoglycan derived, selectin targeting anti-adhesive coating to treat endothelial cell dysfunction. Pharmaceuticals (Basel). 2017;10:36.

6. Proulle V, Furie RA, Merrill-Skoloff G, Furie BC, Furie B. Platelets are required for enhanced activation of the endothelium and fibrinogen in a mouse thrombosis model of APS. Blood. 2014;124:611-622.

7. Capozzi A, Manganelli V, Riitano G, et al. Tissue factor overexpression in platelets of patients with anti-phospholipid syndrome: induction role of anti- $\beta 2-\mathrm{GPI}$ antibodies. Clin Exp Immunol. 2019;196:59-66.

8. Sorice M, Longo A, Capozzi A, et al. Anti-beta2-glycoprotein I antibodies induce monocyte release of tumor necrosis factor alpha and tissue factor by signal transduction pathways involving lipid rafts. Arthritis Rheum. 2007;56:2687-2697.

9. López-Pedrera C, Buendía P, Cuadrado MJ, et al. Antiphospholipid antibodies from patients with the antiphospholipid syndrome induce monocyte tissue factor expression through the simultaneous activation of NF-kappaB/Rel proteins via the p38 mitogenactivated protein kinase pathway, and of the MEK-1/ERK pathway. Arthritis Rheum. 2006;54:301-311.

10. Nadir Y. Heparanase and coagulation-new insights. Rambam Maimonides Med J. 2014;5:e0031.

11. Nadir Y, Brenner B. Heparanase procoagulant activity. Thromb Res. 2012;129:S76-S79.

12. Johnson GB, Brunn GJ, Kodaira Y, Platt JL. Receptor-mediated monitoring of tissue well-being via detection of soluble heparan sulfate by Toll-like receptor 4. J Immunol. 2002;168:5233-5239.

13. Goldberg R, Meirovitz A, Hirshoren N, et al. Versatile role of heparanase in inflammation. Matrix Biol. 2013;32:234-240.

14. Blich M, Golan A, Arvatz G, et al. Macrophage activation by Heparanase is mediated by TLR-2 and TLR-4 and associates with plaque progression. Arterioscler Thromb Vasc Biol. 2013;33: e56-65.

15. Young $E$. The anti-inflammatory effects of heparin and related compounds. Thromb Res. 2008;122:743-752.

16. Goodall KJ, Poon IK, Phipps S, Hulett MD. Soluble Heparan sulfate fragments generated by Heparanase trigger the release of proinflammatory cytokines through TLR-4. PLoS ONE. 2014;9:e109596.

17. Nadir Y, Brenner B, Zetser A, et al. Heparanase induces tissue factor expression in vascular endothelial and cancer cells. J Thromb Haemost. 2006;4:2443-2451.

18. Nadir Y, Brenner B, Gingis-Velitski S, et al. Heparanase induces tissue factor pathway inhibitor expression and extracellular accumulation in endothelial and tumor cells. Thromb Haemost. 2008;99:133-141.

19. Messore A, Madia VN, Pescatori L, et al. Novel symmetrical benzazolyl derivatives endowed with potent anti-heparanase activity. J Med Chem. 2018;61:10834-10859.

20. Pan W, Miao HQ, Xu YJ, et al. 1-[4-(1H-benzoimidazol-2-yl)-p henyl]-3-[4-(1H-benzoimidazol-2- yl)-phenyl]-urea derivatives as small molecule heparanase inhibitors. Bioorg Med Chem Lett. 2006;16:409-412.

21. Madia VN, Messore A, Pescatori L, et al. Novel benzazole derivatives endowed with potent anti-heparanase activity. J Med Chem. 2018;61:6918-6936.

22. Wilson AP. Cytotoxicity and viability assays. In Freshney RI, ed. Animal Cell Culture: A Practical Approach. Oxford: IRL; 1989; 183-216.
23. Temperilli F, Di Franco M, Massimi I, et al. Nonsteroidal antiinflammatory drugs in-vitro and in-vivo treatment and Multidrug Resistance Protein 4 expression in human platelets. Vascul Pharmacol. 2016;76:11-17.

24. Rondina MT, Schwertz HS, Harris E, et al. The septic Milieu triggers expression of spliced tissue factor mRNA in human platelets. $J$ Thromb Haemost. 2011:9:748-758.

25. Ortona E, Capozzi A, Colasanti T, et al. Vimentin/cardiolipin complex as a new antigenic target of the antiphospholipid syndrome. Blood. 2010;116:2960-2967.

26. Farooq AS, Hina D, Ali A, Mildred A, John LF. The presence and release of tissue factor from human platelets. Platelets. 2002:13:247-253.

27. Guarino ML, Massimi I, Mardente S, et al. New platelet functional method for identification of pathogenic antibodies in HIT patients. Platelets. 2017;28:728-730.

28. Panes O, Matus M, Sáez CG, Quiroga T, Pereira J, Mezzano D. Human platelets synthesize and express functional tissue factor. Blood. 2007;109:5242-5250.

29. Raschi E, Testoni C, Bosisio D, Borghi MO, Koike T, Mantovani A Role of the MyD88 transduction signaling pathway in endothelial activation by antiphospholipid antibodies. Blood. 2003;101: 3495-3500.

30. Borghi MO, Raschi E, Grossi C, Chighizola CB, Meroni PL. Toll-like receptor 4 and $\beta 2$ glycoprotein I interaction on endothelial cells. Lupus. 2014;23:1302-1304.

31. Laplante P, Fuentes R, Salem D, et al. Antiphospholipid antibodymediated effects in an arterial model of thrombosis are dependent on Toll-like receptor 4. Lupus. 2016;25:162-166.

32. Andonegui G, Kerfoot SM, McNagny K, Ebbert KVJ, Patel KD, Kubes P. Platelets express functional Toll-like receptor-4. Blood. 2005;106:2417-2423.

33. Berthet J, Damien P, Hamzeh-Cognasse H, Pozzetto B, Garraud O, Cognasse F. Toll-like receptor 4 signal transduction in platelets: novel pathways. Br J Haematol. 2010;151:89-92.

34. Vallance TM, Zeuner MT, Williams HF, Widera D, Vaiyapuri S. Toll-like receptor 4 signalling and its impact on platelet function, thrombosis, and haemostasis. Mediators Inflamm. 2017;2017: 1-13.

35. Lerner I, Hermano E, Zcharia E, et al. Heparanase powers a chronic inflammatory circuit that promotes colitis-associated tumorigenesis in mice. J Clin Invest. 2011;121:1709-1721.

36. Waterman M, Ben-Izhak O, Eliakim R, Groisman G, Vlodavsky I, Ilan $\mathrm{N}$. Heparanase upregulation by colonic epithelium in inflammatory bowel disease. Mod Pathol. 2007:1:8-14.

37. Li RW, Freeman C, Yu D, et al. Dramatic regulation of heparanase activity and angiogenesis gene expression in synovium from patients with rheumatoid arthritis. Arthritis Rheum. 2008;58:1590-1600.

38. Sieve I, Münster-Kühnel AK, Hilfiker-Kleiner D. Regulation and function of endothelial glycocalyx layer in vascular diseases. Vascular Pharmacol. 2018;100:26-33.

39. Vlodavsky I, Singh P, Boyango I, et al. Heparanase: from basic research to therapeutic applications in cancer and inflammation. Drug Resist Update. 2016;29:54-75.

40. Irony-Tur-Sinai M, Vlodavsky I, Ben-Sasson SA, Pinto F, Sicsic C, Brenner T. A synthetic heparin-mimicking polyanionic compound inhibits central nervous system inflammation. J Neurol Sci. 2003;206:49-57.

41. Edovitsky E, Lerner I, Zcharia E, Peretz T, Vlodavsky I, Elkin M. Role of endothelial heparanase in delayed-type hypersensitivity. Blood. 2006;107:3609-3616

42. Floer $M$, Gotte $M$, Wild $M K$, et al. Enoxaparin improves the course of dextran sodium sulfate-induced colitis in syndecan-1-deficient mice. Am J Pathol. 2010;176:146-157. 
43. Kato H. Regulation of functions of vascular wall cells by tissue factor pathway inhibitor: basic and clinical aspects. Arterioscler Thromb Vasc Biol. 2002;22:539-548.

\section{SUPPORTING INFORMATION}

Additional supporting information may be found online in the Supporting Information section.
How to cite this article: Capozzi A, Riitano G, Recalchi S, et al. Effect of heparanase inhibitor on tissue factor overexpression in platelets and endothelial cells induced by anti-ß2-GPI antibodies. J Thromb Haemost. 2021;19:23022313. https://doi.org/10.1111/jth.15417 